\title{
Das Labyrinth - ein Tanz?
}

Am Beispiel des Labyrinthtanzes wollen wir uns den Einsatz des traditionellen griechischen Tanzes im psychomotorischen Kontext genauer ansehen. Dabei liegt unser Augenmerk auf den kognitiven, emotionalen, sozialen und motorischen Impulsen der traditionellen griechischen Tänze im Kontext einer ganzheitlichen Gesundheitsbildung in der Gegenwart.

\section{Historische und soziokulturelle Einordnung} Das Labyrinth - ein Tanz? Jahrtausende alte griechische Chroniken stützen diese These. Dafür sprechen kreisförmige Tanzplätze, Darstellungen von Bewegungen, in denen sich der Glaube früher Kulturen an die unendliche Spirale von Leben, Tod und Wiedergeburt spiegelt.

Wir finden Tanz als Bewegung in Form des Ur-Labyrinthes. "Sehr vieles spricht für die Annahme«, dass sich das Ur-Labyrinth "zunächst als Gruppentanz manifestiert hat und dass die Tänzerkette die Wegbahnen nachgeschritten ist, die auch auf dem Täfelchen von Pylos dargestellt sind « (Kern 1999, 18).

Dem Labyrinthtanz wird bis heute eine kraftvolle Wirkung zugeschrieben und er galt bereits im antiken Epidauros als ein wichtiges Element zur Gesundheitsbildung. In der Antike war der Labyrinthtanz als Labyrinthos oder Géranos (Kranichtanz) bekannt und wurde erstmals bei Homer erwähnt (Homer 1979, 590-602). Plutarch zufolge tanzte Theseus auf Delos den Géranos, den ihn Ariadne zuvor gelehrt hatte. Bis heute überlebte der Tanz Géranos unter dem Namen Tsakónikos (Stratou 1966, 20f).

\section{Interpretationsansatz und psychomotorische Einordnung}

Das Labyrinth gehört zu den ältesten Symbolen der Menschheit. Es zu beschreiten bedeutet, aus der gewohnten Bahn geführt zu werden. Das Labyrinth bringt Wendungen, Staunen, vielleicht auch Irritation und ermöglicht immer wieder neue Orientierung im Raum (Abb.1).

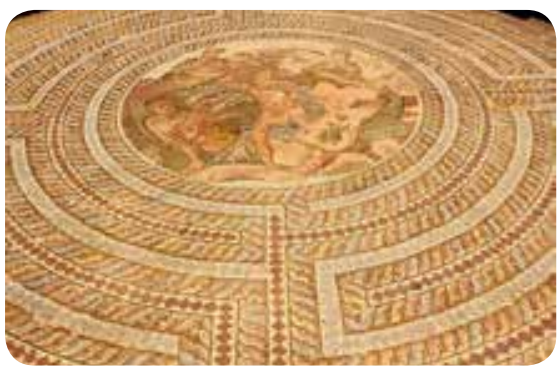

Abb. 1: Mosaik mit Theseus, Minotaurus, Labyrinthos, Ariadne und Kreta umgeben vom Labyrinth. Archäologischer Park Paphos. Haus des Theseus.

(Foto: Thomas Chamalidis)

In sein Zentrum zu gelangen heißt, ins Gleichgewicht zu kommen, um aus der Kraft der Mitte wieder hinausgehen zu können. Eine beständige mäandrierende Bewegung bildet den Weg hinein und wieder heraus und hilft uns, in den Rhythmus und Zyklus des Lebens zurückzufinden. Zusätzlich zur mäandrierenden Bewegung erfahren wir einen rhythmisierten motorischen Impuls durch eine wiederkehrende Tanzbewegung und eine sich wiederholende Schrittabfolge.

Im Tanz sind wir dabei eingebunden in die Gemeinschaft und werden von ihr gehalten (Abb. 2). Nicht nur nehmen wir unseren Platz in der Gemeinschaft ein und entwickeln dabei soziale Kompetenzen im Miteinander, sondern gehen auch eine haptische Verbindung mit unseren Nachbarn ein. Diese haptische
Verbindung kann in der Praxis durch einen Stoff ergänzt werden. Es eignet sich dazu ein roter Faden, der den Faden der Ariadne symbolisiert. Der Vollständigkeit halber sei darauf hingewiesen, dass dieses Element im traditionellen Kontext zwar nicht zu finden ist, jedoch erwähnt Plutarch derartiges im Rahmen seiner Beschreibung des Géranos.

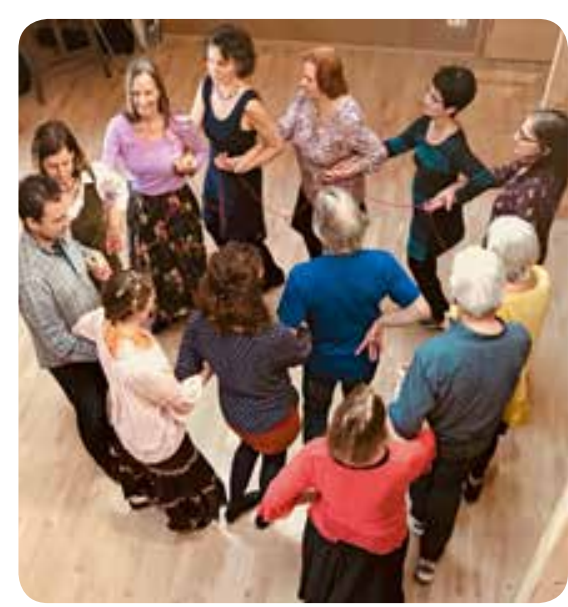

Abb. 2: Tanzkreis "Tsakonikos" (Foto: Savina Chamalidis)

Der Tanzkreis wird vom ersten Tänzer bzw. von der ersten Tänzerin angeführt. Die erste Person übernimmt die Rolle des Theseus und damit Verantwortung für die Gruppe. Das Selbstbewusstsein und Verantwortungsgefühl der Person werden gestärkt, indem sie die Gruppe in Pendelbewegungen sicher im Raum führt. Sie entwickelt dabei ein ausgeprägtes Raumgefühl und ihre Eigenund Fremdwahrnehmung werden gefördert.

Psychomotorische Impulse des Labyrinthtanzes im Überblick:

\footnotetext{
- haptische und stoffliche Verbindung,

- soziale Kompetenz,

motorische Fähigkeit,
} 
- Rhythmuserfahrung,

- gruppendynamischer Prozess,

- Stärkung des Gemeinschaftssinns,

- Selbstbewusstsein und Verantwortungsgefühl in der Gruppe (»Theseus«),

- Förderung der propriozeptiven Wahrnehmung (Tiefensensibilität) im Sinne einer Schulung des Lagesinns bzw. Positionssinns (Position des Körpers im Raum und Stellung der Gelenke und des Kopfes), der Ausbildung des Kraftsinns (Information über den Spannungszustand von Muskeln und Sehnen) und des Bewegungssinns, durch den eine Bewegungsempfindung und das Erkennen der Bewegungsrichtung ermöglicht wird,

- Eigen- und Fremdwahrnehmung.

\section{Stundenaufbau}

Der Stundenaufbau gliedert sich in sieben Schritte:

Schritt 1: Mythologische Einordnung des Labyrinththemas (Theseus und Ariadne)

\section{- Identifikation mit dem Thema}

Schritt 2: Malen des kretischen Labyrinthes (Abb.3)

- Identifikation mit der Form des Labyrinthes

- Schulung der Feinmotorik

- Förderung der visomotorischen Koordination

- Erfolgserlebnis durch haptischmotorische Selbstkontrolle beim Durchfahren des gemalten Labyrinthes mit dem Finger

Schritt 3: Vorstellung der Tanzbewegung und Handfassung (Lehrvideo auf Anfrage)

- Anregung der visomotorischen Koordination
Schritt 4: Tanzen im Kreis mit Musik (Musikdatei auf Anfrage)

- Förderung der propriorezeptiven Wahrnehmung

- Erprobung motorischer Fähigkeiten

- Rhythmuserfahrung

- Erfahrung haptischer Impulse

Schritt 5: Tanzen mäandrierend im Raum

- Bildung sozialer Kompetenz

- Stärkung des Gemeinschaftssinns/ gruppendynamischer Prozess

- Schulung von Verantwortungsgefühl und Selbstbewusstsein

- Ausbildung von Eigen- und Fremdwahrnehmung

Schritt 6: Wiederholung

- bei jeder Wiederholung besteht die Möglichkeit, die vordere Person zu wechseln

Schritt 7: Reflexion / Impuls

[ »Das Labyrinth ist für mich ...»

n »Das Labyrinth bedeutet für mich ..."

[ "Das Labyrinth steht für mich für..."

- »Wenn ich Labyrinth höre, denke ich an ..."

" »Das Labyrinth kann ..."

" "Das Labyrinth fühle ich ...»

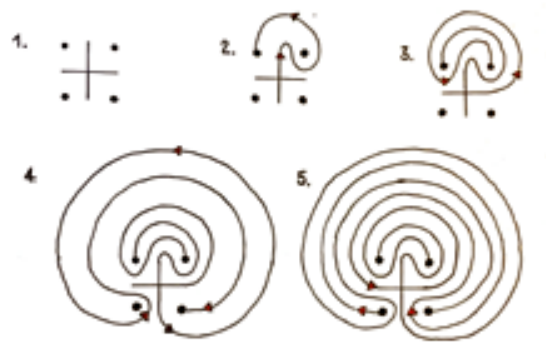

Abb. 3: Malvorlage "Labyrinth" (ㄷ) Labbé, www.labbe.de)
Tanzbeschreibung »Tsakonikos»

Herkunft: Tsakonia in Arkadien (Ost-Peloponnes)

Takt: 5/8 (langsam) oder 5/4 (schnell)

Handfassung: eingehakt, rechte Hand der linken Person greift die linke Hand der rechten Person

Tanzform: offener Halbkreis/Spirale / Mäander

Einsatz: mit Beginn des melodischen Bogens

Grundschritte: 5 Schritte im 1. Teil und 5 Schritte im 2. Teil

\section{Teil}

rechts in Tanzrichtung rechts $\rightarrow$ links in Tanzrichtung rechts $\rightarrow$ rechts in Tanzrichtung rechts $\rightarrow$ links in Tanzrichtung rechts $\rightarrow$ rechter Fußballen ohne Gewicht neben linker Ferse $\rightarrow$

\section{Teil}

rechts in Tanzrichtung rechts $\rightarrow$ links in Tanzrichtung rechts $\rightarrow$ rechts in Tanzrichtung rechts $\rightarrow$ rechts hüpft auf der Stelle links setzt in Tanzrichtung auf $\rightarrow$

\section{(1) Literatur}

Homer (1979): Ilias, Übersetzung Roland Hampe. Philipp Reclam jun., Stuttgart Kern, H. (1999): Labyrinthe. Erscheinungsformen und Deutungen. 5000 Jahre Gegenwart eines Urbilds. Prestel Verlag, München

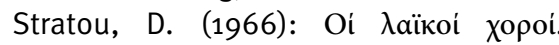

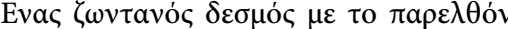
(Greek Dances - Our Living Link With Antiquity). Dora Stratou, Athen

Videolink »Tsakonikos«: https://www. youtube.com/watch?v=05uj6Y2l6Yo

Savina Chamalidis

info@estia-sfakia.gr

DOI 10.2378/ mot2019.arto7d 